Journal of

\section{CRYPTOLOGY}

(C) 2007 International Association for Cryptologic Research

\title{
RSA-Based Undeniable Signatures
}

\author{
Rosario Gennaro and Tal Rabin \\ IBM T.J. Watson Research Center, \\ PO Box 704, Yorktown Heights, NY 10598, U.S.A. \\ \{rosario,talr\}@watson.ibm.com \\ Hugo Krawczyk \\ IBM T.J. Watson Research Center, \\ PO Box 704, Yorktown Heights, NY 10598, U.S.A. \\ and \\ Department of Electrical Engineering, Technion, \\ Haifa 32000, Israel \\ hugo@ee.technion.ac.il
}

Erratum: The online contents page of the original article (DOI: 10.1007/s001450010001) was inadvertently published with only the first author appearing. The correct version appears above. The publisher regrets and apologizes for the error. 